\title{
Stereoselective Mn-Mediated Coupling of Functionalized lodides and Hydrazones: A Synthetic Entry to the Tubulysin $\gamma$-Amino Acids
}

Gregory K. Friestad, * Jean-Charles Marié, and Amy M. Deveau

Department of Chemistry, University of Vermont, Burlington, VT 05405

gregory.friestad@uvm.edu

\section{Supporting Information}

Contents:

S1-S7 Procedures and characterization data for new compounds

S8 NMR spectra for configurational assignment of compound $\mathbf{1 0}$ 
Materials and Methods. Reactions employed oven- or flame- dried glassware under a nitrogen atmosphere unless otherwise noted. Tetrahydrofuran (THF), toluene and benzene were distilled over sodium/benzophenone ketyl and dichloromethane $\left(\mathrm{CH}_{2} \mathrm{Cl}_{2}\right)$ from $\mathrm{CaH}_{2}$ under a nitrogen atmosphere. Nitrogen was passed successively through columns of anhydrous $\mathrm{CaSO}_{4}$ and R3-11 catalyst (SchweizerHall, South Plainfield, NJ) for removal of water and oxygen respectively. All other materials were used as received from Aldrich or purified by standard procedures. Thin layer chromatography (TLC) employed glass $0.25 \mathrm{~mm}$ silica gel plates with UV indicator. Flash chromatography columns were packed with 230-400 mesh silica gel as slurry in the hexane. Gradient flash chromatography was conducted by adsorption of the crude mixture on silica gel, packing over a short pad of clean silica gel as a slurry in hexane, and eluting with a continuous gradient from hexane to the indicated solvent. Melting points were determined on a Meltemp apparatus and are uncorrected. Proton and carbon NMR data were obtained with a Bruker ARX 500 spectrometer. Infrared spectra were recorded with a Perkin-Elmer 2000 FT-IR spectrophotometer. Optical rotations were determined using a Rudolph Research Autopol IV Polarimeter. Low resolution mass spectra were obtained with a Finnegan 4610 quadrupole spectrometer or a Hewlett Packard 5988 GCMS. Combustion analyses were performed by Atlantic Microlab (Norcross, GA). Diastereomer ratios were determined by integration of ${ }^{1} \mathrm{H}$ NMR spectra.

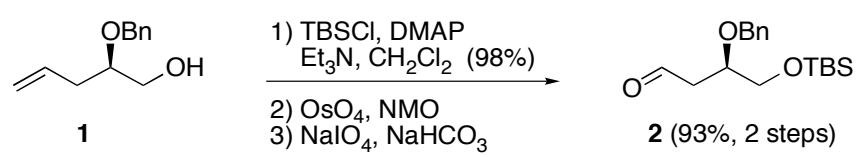

Aldehyde 2. To a solution of pentenol $1(60 \mathrm{mg}, 0.31 \mathrm{mmol})$ in $\mathrm{CH}_{2} \mathrm{Cl}_{2}(1 \mathrm{~mL}), \mathrm{NEt}_{3}(86 \mu \mathrm{L}, 0.62$ mmol) and DMAP (4 mg, $0.03 \mathrm{mmol}$ ) were added at room temperature. The solution was stirred at $0^{\circ} \mathrm{C}$ for 5 minutes and TBSCl (49 mg, $0.33 \mathrm{mmole}$ ) was added at once. After one hour, the reaction mixture was allowed to warm to room temperature, stirred overnight, and then diluted with $\mathrm{CH}_{2} \mathrm{Cl}_{2}(15 \mathrm{~mL})$. The mixture was extracted with sat. $\mathrm{NH}_{4} \mathrm{Cl}$, washed with brine and dried over $\mathrm{MgSO}_{4}$. Concentration and flash column chromatography $\left(\mathrm{Hex} \rightarrow 90: 10 \mathrm{Hex}_{2} \mathrm{Et}_{2} \mathrm{O}\right)$ afforded the silyl ether as a colorless oil (94 mg, 98\% yield); $[\alpha]_{\mathrm{D}}^{20}+6.7\left(c\right.$ 2.45, $\left.\mathrm{CHCl}_{3}\right)$; IR (film) 2954, 1471, 1256, 1110, 913, 837, $776 \mathrm{~cm}^{-1} ;{ }^{1} \mathrm{H}$ NMR (500 MHz, $\left.\mathrm{CDCl}_{3}\right) \delta$ 7.37-7.27 (m, 5H), 5.92-5.84 (m, 1H), 5.12 (dd, $\left.J=17.1,1.5 \mathrm{~Hz}, 1 \mathrm{H}\right), 5.07$ $(\mathrm{dt}, J=10.2,1.0 \mathrm{~Hz}, 1 \mathrm{H}), 4.66(\mathrm{ABq}, J=11.8 \mathrm{~Hz}, \Delta v=23.9 \mathrm{~Hz}, 2 \mathrm{H}), 3.67\left(\mathrm{ABX}, J_{\mathrm{AB}}=10.5 \mathrm{~Hz}, J_{\mathrm{AX}}=\right.$ $\left.5.9 \mathrm{~Hz}, J_{\mathrm{BX}}=5.2 \mathrm{~Hz}, \Delta v=30.5 \mathrm{~Hz}, 2 \mathrm{H}\right), 3.64(\mathrm{dd}, J=10.5,5.2 \mathrm{~Hz}, 1 \mathrm{H}), 3.55$ (dddd, apparent quintet, $J$ $=5.8 \mathrm{~Hz}, 1 \mathrm{H}), 2.42-2.36(\mathrm{~m}, 1 \mathrm{H}), 2.34-2.29(\mathrm{~m}, 1 \mathrm{H}), 0.93(\mathrm{~s}, 9 \mathrm{H}), 0.08(\mathrm{~s}, 6 \mathrm{H}) ;{ }^{13} \mathrm{C} \mathrm{NMR}(125 \mathrm{MHz}$, $\left.\mathrm{CDCl}_{3}\right) \delta 139.0,134.9,128.2(2 \mathrm{C}), 127.6(2 \mathrm{C}), 127.4,116.8,79.5,72.0,65.1,36.0,25.9(3 \mathrm{C}), 18.2,-5.4$

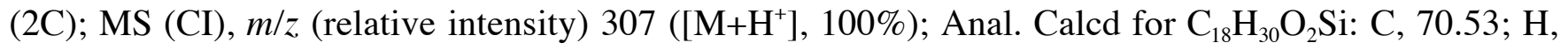
9.87. Found: C, 70.74; H, 9.98 .

To a solution of the silyl ether obtained as described above $(86 \mathrm{mg}, 0.28 \mathrm{mmol})$ in a mixture of THF $(2.5 \mathrm{~mL})$, water $(0.5 \mathrm{~mL})$ and tert-butanol $(5 \mathrm{~mL}), \mathrm{NMO}(49 \mathrm{mg}, 0.42 \mathrm{mmol})$ and $\mathrm{OsO}_{4}(2.5 \%$ wt in tertbutanol, $150 \mu \mathrm{L}, 0.01 \mathrm{mmol}$ ) were added at $0{ }^{\circ} \mathrm{C}$. The reaction mixture was allowed to warm to room temperature, stirred overnight and quenched with sat. $\mathrm{NaHSO}_{3}(10 \mathrm{~mL})$. After $1 \mathrm{~h}$ at room temperature, the crude mixture was poured into water and extracted with EtOAc. The organic phase was washed with brine and dried with $\mathrm{MgSO}_{4}$. Concentration afforded a diol which was used without further purification.

To a solution of the diol obtained as described above (ca. $0.28 \mathrm{mmol})$ in water $(2 \mathrm{~mL}), \mathrm{NaHCO}_{3}(47$ $\mathrm{mg}, 0.56 \mathrm{mmol}$ ) and $\mathrm{NaIO}_{4}$ (as a solution in $1 \mathrm{~mL}$ of water, $120 \mathrm{mg}, 0.56 \mathrm{mmol}$ ) were successively added at $0^{\circ} \mathrm{C}$ with vigorous stirring. After one hour, the mixture was allowed to warm to room temperature, stirred overnight and poured into EtOAc. The organic phase was washed with brine and dried over $\mathrm{MgSO}_{4}$. Concentration and flash chromatography (5:1 Hex/EtOAc) afforded the aldehyde 2 as a colorless oil $\left(80 \mathrm{mg}, 93 \%\right.$ yield); $[\alpha]_{\mathrm{D}}^{20}+23.1$ (c 1.30, $\left.\mathrm{CHCl}_{3}\right)$; IR (film) 2954, 2929, 2857, 1727, $1471,1463,1255,1095,1028,837,777,736,697 \mathrm{~cm}^{-1} ;{ }^{1} \mathrm{H}$ NMR $\left(500 \mathrm{MHz}, \mathrm{CDCl}_{3}\right) \delta 9.79(\mathrm{t}, J=2.0$ 
$\mathrm{Hz}, 1 \mathrm{H}), 7.36-7.28(\mathrm{~m}, 5 \mathrm{H}), 4.64(\mathrm{ABq}, J=11.7 \mathrm{~Hz}, \Delta v=34.3 \mathrm{~Hz}, 2 \mathrm{H}), 4.01$ (dddd, apparent quintet, $J$ $=5.6 \mathrm{~Hz}, 1 \mathrm{H}), 3.77(\mathrm{dd}, J=10.3,5.0 \mathrm{~Hz}, 1 \mathrm{H}), 3.64(\mathrm{dd}, J=10.4,5.9 \mathrm{~Hz}, 1 \mathrm{H}), 2.67-2.65(\mathrm{~m}, 2 \mathrm{H}), 0.90$ (s, 9H), 0.07 (s, 3H), $0.06(\mathrm{~s}, 3 \mathrm{H}) ;{ }^{13} \mathrm{C} \mathrm{NMR}\left(125 \mathrm{MHz}, \mathrm{CDCl}_{3}\right.$ ) $\delta 200.8,138.2,128.4(2 \mathrm{C}), 127.7$ (3C), 75.1, 72.1, 64.6, 46.3, 25.8 (3C), 18.2, -5.5 (2C); MS (CI), m/z (relative intensity) $309\left(\left[\mathrm{M}+\mathrm{H}^{+}\right], 76 \%\right)$; Anal. Calcd for $\mathrm{C}_{17} \mathrm{H}_{28} \mathrm{O}_{3}$ Si: C, 66.19; H, 9.15. Found: C, 66.17; H, 9.23.

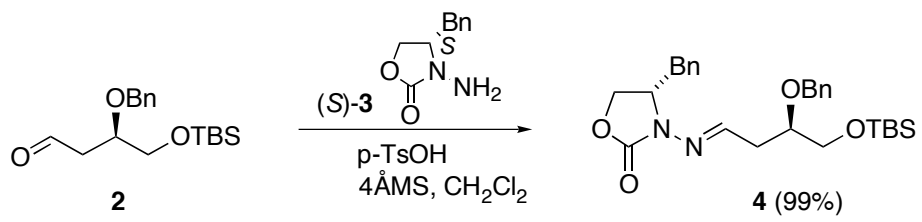

Hydrazone 4 (General Procedure A). To a mixture of $N$-amino oxazolidinone $(S)-3^{1}(75 \mathrm{mg}, 0.39$ mmol), $p$-toluenesulfonic acid (1 mg) and powdered activated $4 \AA$ molecular sieves $(25 \mathrm{mg})$ in $\mathrm{CH}_{2} \mathrm{Cl}_{2}(1$ $\mathrm{mL})$ was added a solution of aldehyde $2(60 \mathrm{mg}, 0.19 \mathrm{mmol})$ in $\mathrm{CH}_{2} \mathrm{Cl}_{2}(1 \mathrm{~mL})$ via cannula. The solution was allowed to stir overnight at room temperature. Filtration through Celite, concentration, and flash chromatography (99:1 Hex/NEt $\left.\rightarrow 70: 30: 1 \mathrm{Hex} / \mathrm{EtOAc} / \mathrm{NEt}_{3}\right)$ afforded 4 as a pale yellow oil (93 mg, 99\% yield); $[\alpha]_{\mathrm{D}}^{20}+13.6$ (c 1.95, $\mathrm{CHCl}_{3}$ ); IR (film) 2953, 2928, 2856, 1773, 1757, 1497, 1471, 1454, 1400, 1360, 1251, 1211, 1093, 837, 778, 743, $699 \mathrm{~cm}^{-1} ;{ }^{1} \mathrm{H}$ NMR (500 MHz, $\left.\mathrm{CDCl}_{3}\right) \delta 8.07$ (t, J=5.6 $\mathrm{Hz}, 1 \mathrm{H}), 7.36-7.25(\mathrm{~m}, 8 \mathrm{H}), 7.12(\mathrm{~m}, 2 \mathrm{H}), 4.67(\mathrm{ABq}, J=11.7 \mathrm{~Hz}, \Delta v=44.5 \mathrm{~Hz}, 2 \mathrm{H}), 4.33-4.27(\mathrm{~m}$, $1 \mathrm{H}), 4.22(\mathrm{dd}, J=8.6,8.6 \mathrm{~Hz}, 1 \mathrm{H}), 4.09(\mathrm{dd}, J=8.8,5.3 \mathrm{~Hz}, 1 \mathrm{H}), 3.82-3.76(\mathrm{~m}, 2 \mathrm{H}), 3.72-3.69(\mathrm{~m}, 1 \mathrm{H})$, $3.20(\mathrm{dd}, J=13.8,3.5 \mathrm{~Hz}, 1 \mathrm{H}), 2.74(\mathrm{dd}, J=13.7,9.0 \mathrm{~Hz}, 1 \mathrm{H}), 2.70(\mathrm{dd}, J=5.3,4.8 \mathrm{~Hz}, 1 \mathrm{H}), 2.67-2.61$ $(\mathrm{m}, 1 \mathrm{H}), 0.92(\mathrm{~s}, 9 \mathrm{H}), 0.08(\mathrm{~s}, 3 \mathrm{H}), 0.08(\mathrm{~s}, 3 \mathrm{H}) ;{ }^{13} \mathrm{C} \mathrm{NMR}\left(125 \mathrm{MHz}, \mathrm{CDCl}_{3}\right) \delta$ 154.3, 153.5, 138.5, 135.2, 129.2 (2C), 128.9 (2C), 128.3 (2C), 127.7 (2C), 127.6, 127.2, 77.7, 72.1, 65.6, 65.1, 57.4, 36.9, 35.8, 25.9 (3C), 18.3, -5.4 (2C); MS (CI), $m / z$ (relative intensity) 484 ([M+H $\left.\mathrm{H}^{+}\right], 36 \%$ ); Anal. Calcd for $\mathrm{C}_{27} \mathrm{H}_{38} \mathrm{~N}_{2} \mathrm{O}_{4} \mathrm{Si}: \mathrm{C}, 67.18 ; \mathrm{H}, 5.80 ; \mathrm{N}, 7.94$. Found: C, 67.40; H, 5.85; N, 8.08.

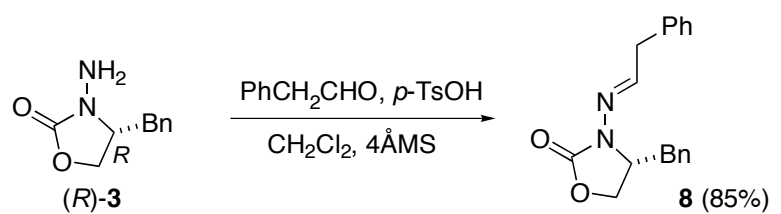

Hydrazone 8. From freshly distilled phenyl-acetaldehyde $(263 \mu \mathrm{L}, 2.25 \mathrm{mmol})$ and $(R)-3$-Amino-4phenylmethyl-2-oxazolidinone ${ }^{2}(865 \mathrm{mg}, 4.50 \mathrm{mmol})$, following the General Procedure A, the hydrazone (8) was obtained as a white solid $(563 \mathrm{mg}, 85 \%$ yield) which could be recrystallized from 99:1 Hex $/ \mathrm{CH}_{2} \mathrm{Cl}_{2} ; \mathrm{mp} 64-66^{\circ} \mathrm{C} ;[\alpha]_{\mathrm{D}}^{20}+4.1$ (c 0.51, $\mathrm{CHCl}_{3}$ ); IR (film) 3061, 3027, 2917, 1768, 1603, 1496, 1453, 1402, 1352, 1212, 1083, 1030, 1005, 752, $701 \mathrm{~cm}^{-1} ;{ }^{1} \mathrm{H}$ NMR $\left(500 \mathrm{MHz}, \mathrm{CDCl}_{3}\right) \delta 8.11(\mathrm{t}, J$ $=5.9 \mathrm{~Hz}, 1 \mathrm{H}), 7.37-7.26(\mathrm{~m}, 8 \mathrm{H}), 7.16(\mathrm{~m}, 2 \mathrm{H}), 4.40-4.35(\mathrm{~m}, 1 \mathrm{H}), 4.23(\mathrm{dd}, J=8.7,8.7 \mathrm{~Hz}, 1 \mathrm{H}), 4.09$ $(\mathrm{dd}, J=8.9,5.3 \mathrm{~Hz}, 1 \mathrm{H}), 3.73(\mathrm{~d}, J=5.8 \mathrm{~Hz}, 2 \mathrm{H}), 3.22(\mathrm{dd}, J=13.9,3.7 \mathrm{~Hz}, 1 \mathrm{H}), 2.83(\mathrm{dd}, J=13.9$, $8.7 \mathrm{~Hz}, 1 \mathrm{H}) ;{ }^{13} \mathrm{C} \mathrm{NMR}\left(125 \mathrm{MHz}, \mathrm{CDCl}_{3}\right) \delta 154.3,153.7,136.2,135.2,129.3$ (2C), 128.9 (2C), 128.8 (2C), 128.7 (2C), 127.2, 126.9, 65.7, 57.5, 39.9, 37.1; MS (CI), m/z (relative intensity) $295\left(\left[\mathrm{M}+\mathrm{H}^{+}\right]\right.$, 100\%); Anal. Calcd for $\mathrm{C}_{18} \mathrm{H}_{18} \mathrm{~N}_{2} \mathrm{O}_{2}$ : C, 73.45; H, 6.16; N, 9.52. Found: C, 73.38; H, 6.14; N, 9.48.

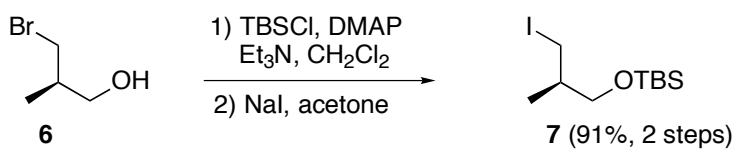

Iodide 7. ${ }^{3}$ To a solution of commercially available bromide $6(1.00 \mathrm{~mL}, 9.68 \mathrm{mmol})$ in $\mathrm{CH}_{2} \mathrm{Cl}_{2}(30$ $\mathrm{mL})$, at $0^{\circ} \mathrm{C}, \mathrm{NEt}_{3}(2.70 \mathrm{~mL}, 19.36 \mathrm{mmol})$, DMAP $(118 \mathrm{mg}, 0.97 \mathrm{mmol})$ and TBSCl $(1.53 \mathrm{~g}, 10.16$ 
mmol) were successively added. After $1 \mathrm{~h}$, the reaction mixture was warmed to ambient temperature overnight, then partitioned between $\mathrm{CH}_{2} \mathrm{Cl}_{2}$ and sat. $\mathrm{NH}_{4} \mathrm{Cl}$. The organic phase was dried over $\mathrm{Na}_{2} \mathrm{SO}_{4}$ and concentrated to afford the silyl ether as a pale yellow oil which was used without further purification.

To a solution of the silyl bromide obtained as described above $(2.59 \mathrm{~g}, 9.68 \mathrm{mmol})$ in dry acetone (30 $\mathrm{mL})$ was added $\mathrm{NaI}(4.35 \mathrm{~g}, 29.10 \mathrm{mmol})$ in one portion. The solution was stirred for 2 days at $50^{\circ} \mathrm{C}$, poured into water and extracted with EtOAc. The organic phase was washed with brine and dried over $\mathrm{MgSO}_{4}$. Concentration and vacuum distillation afforded iodide 7 as a colorless oil $(2.83 \mathrm{~g}$, 93\% yield over 2 steps); bp $66-67^{\circ} \mathrm{C}(0.80 \mathrm{mmHg}) ;[\alpha]_{\mathrm{D}}^{20}-10.2\left(\right.$ c $\left.7.90, \mathrm{CHCl}_{3}\right)$.

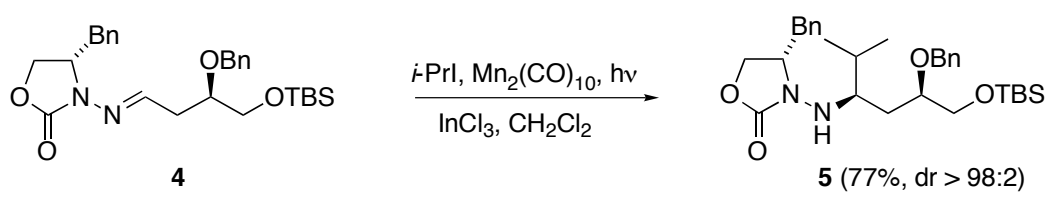

Hydrazine 5 (General Procedure B: Mn-Mediated Addition to $\boldsymbol{N}$-Acylhydrazones. ${ }^{4}$ ). To a suspension of $\mathrm{InCl}_{3}(243 \mathrm{mg}, 1.10 \mathrm{mmol})$ in $\mathrm{CH}_{2} \mathrm{Cl}_{2}(3 \mathrm{~mL})$ was added hydrazone $4(241 \mathrm{mg}, 0.50$ $\mathrm{mmol}$ ) in $\mathrm{CH}_{2} \mathrm{Cl}_{2}(2 \mathrm{~mL})$. The mixture was stirred for $1 \mathrm{~h}$ at room temperature, then $i$-PrI (filtered over alumina pad, $400 \mu \mathrm{L}, 4.00 \mathrm{mmol})$ and $\mathrm{Mn}_{2}(\mathrm{CO})_{10}($ Strem, $195 \mathrm{mg}, 0.50 \mathrm{mmol}$ ) were added successively. Using a Rayonet photochemical reactor, ultraviolet irradiation $(300 \mathrm{~nm})$ was supplied for $18-20 \mathrm{~h}$; the ambient temperature inside the irradiation chamber reached ca $35^{\circ} \mathrm{C}$. Addition of $\mathrm{NEt}_{3}(0.70 \mathrm{~mL}, 5.00$ mmol) and vigorous stirring for 3 hours, followed by filtration through silica gel ( $\mathrm{Et}_{2} \mathrm{O}$ eluent), concentration, and flash chromatography (Hex $\rightarrow 3 / 1 \mathrm{Hex}:$ EtOAc) afforded recovered starting material $(17 \mathrm{mg}, 7 \%)$ and hydrazine 5 as a colorless oil $(204 \mathrm{mg}, 77 \%$ yield, d.r. $>98: 2) ;[\alpha]_{\mathrm{D}}^{20}+57.1(c 7.20$, $\mathrm{CHCl}_{3}$ ); IR (film) 3287, 2955, 2929, 2857, 1761, 1496, 1472, 1456, 1394, 1359, 1251, 1088, 836, 776, 737, $699 \mathrm{~cm}^{-1} ;{ }^{1} \mathrm{H}$ NMR $\left(500 \mathrm{MHz}, \mathrm{CDCl}_{3}\right) \delta 7.35-7.24(\mathrm{~m}, 8 \mathrm{H}), 7.08(\mathrm{~m}, 2 \mathrm{H}), 4.77(\mathrm{~d}, J=11.8 \mathrm{~Hz}$, $1 \mathrm{H}), 4.64(\mathrm{~d}, J=11.8 \mathrm{~Hz}, 1 \mathrm{H}), 4.35$ (br s, $1 \mathrm{H}), 4.05(\mathrm{dd}, J=8.7,7.7 \mathrm{~Hz}, 1 \mathrm{H}), 3.97$ (dd, $J=8.8,5.3 \mathrm{~Hz}$, $1 \mathrm{H}), 3.84-3.77(\mathrm{~m}, 3 \mathrm{H}), 3.67(\mathrm{dd}, J=9.8,5.4 \mathrm{~Hz}, 1 \mathrm{H}), 3.25(\mathrm{dd}, J=13.5,3.4 \mathrm{~Hz}, 1 \mathrm{H}), 3.13(\mathrm{br} \mathrm{d}, J=$ $8.7 \mathrm{~Hz}, 1 \mathrm{H}), 2.54(\mathrm{dd}, J=13.4,10.0 \mathrm{~Hz}, 1 \mathrm{H}), 1.96(\mathrm{~m}$, apparent septet of doublets, $J=6.9,3.1 \mathrm{~Hz}, 1 \mathrm{H})$, $1.63(\mathrm{ddd}, J=14.9,7.5,2.5 \mathrm{~Hz}, 1 \mathrm{H}), 1.53(\mathrm{ddd}, J=14.7,9.4,3.4 \mathrm{~Hz}, 1 \mathrm{H}), 0.97(\mathrm{~d}, J=6.8 \mathrm{~Hz}, 3 \mathrm{H}), 0.95$ $(\mathrm{d}, J=7.0 \mathrm{~Hz}, 3 \mathrm{H}), 0.92(\mathrm{~s}, 9 \mathrm{H}), 0.09(\mathrm{~s}, 3 \mathrm{H}), 0.08(\mathrm{~s}, 3 \mathrm{H}) ;{ }^{13} \mathrm{C} \mathrm{NMR}\left(125 \mathrm{MHz}, \mathrm{CDCl}_{3}\right) \delta 158.6,138.9$, 135.9, 129.0 (2C), 128.8 (2C), 128.3 (2C), 127.5 (2C), 127.4, 126.9, 78.2, 72.4, 65.7, 65.2, 60.0, 58.4, 36.7, 30.0, 28.7, 25.9 (3C), 19.1, 18.2, 15.9, -5.4 (2C); Anal. Calcd for $\mathrm{C}_{30} \mathrm{H}_{46} \mathrm{~N}_{2} \mathrm{O}_{4} \mathrm{Si}$ : C, 68.40; H, 8.80; N, 5.32. Found: C, 68.36; H, 8.88; N, 5.45.
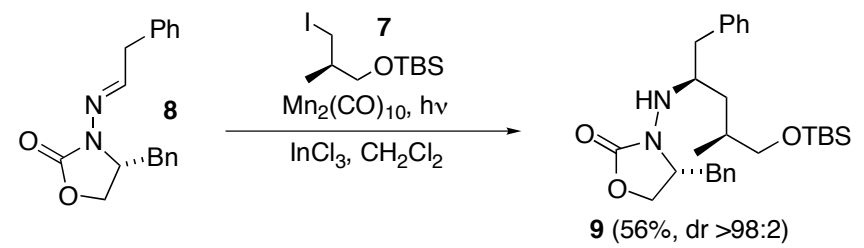

Hydrazine 9. From hydrazone 8 (73 mg, $0.25 \mathrm{mmol}$ ) and freshly distilled iodide (7) (393 $\mathrm{mg}, 1.25$ mmol), following the General Procedure (B), was obtained the starting hydrazone 8 (6 $\mathrm{mg}, 8 \%)$ and hydrazine 9 as a colorless oil (67 mg, 56\% yield, $\mathrm{dr}>98: 2) ;[\alpha]_{\mathrm{D}}^{20}-32.0\left(c 5.50, \mathrm{CHCl}_{3}\right)$; IR (film) 3287 , 3028, 2953, 2929, 2857, 1758, 1496, 1456, 1397, 1363, 1251, 1091, 1031, 839, 776, 744, $700 \mathrm{~cm}^{-1} ;{ }^{1} \mathrm{H}$ NMR (500 MHz, $\left.\mathrm{CDCl}_{3}\right) \delta$ 7.31-7.20 (m, 8H), $7.06(\mathrm{~m}, 2 \mathrm{H}), 4.10$ (br s, 1H), 3.93-3.87 (m, 2H), 3.63$3.59(\mathrm{~m}, 1 \mathrm{H}), 3.51-3.44(\mathrm{~m}, 3 \mathrm{H}), 3.13(\mathrm{dd}, J=13.4,3.2 \mathrm{~Hz}, 1 \mathrm{H}), 2.78\left(\mathrm{ABX}, J_{\mathrm{AB}}=14.0 \mathrm{~Hz}, J_{\mathrm{AX}}=5.5\right.$ $\left.\mathrm{Hz}, J_{\mathrm{BX}}=7.6 \mathrm{~Hz}, \Delta v=46.0 \mathrm{~Hz}, 2 \mathrm{H}\right), 2.46(\mathrm{dd}, J=13.3,10.2 \mathrm{~Hz}, 1 \mathrm{H}), 1.87-1.79(\mathrm{~m}, 1 \mathrm{H}), 1.61(\mathrm{ddd}, J=$ 
13.5, 6.7, $6.4 \mathrm{~Hz}, 1 \mathrm{H}), 1.34$ (ddd, J = 13.9, 7.6, $6.3 \mathrm{~Hz}, 1 \mathrm{H}), 0.95$ (d, J = 6.7 Hz, 3H), 0.89 (s, 9H), 0.04 (s, 3H), $0.03(\mathrm{~s}, 3 \mathrm{H}) ;{ }^{13} \mathrm{C} \mathrm{NMR}\left(125 \mathrm{MHz}, \mathrm{CDCl}_{3}\right) \delta 158.4,139.3,135.9,129.2(2 \mathrm{C}), 129.0$ (2C), 128.8 (2C), 128.4 (2C), 126.9, 126.2, 67.7, 65.6, 59.2, 58.5, 40.4, 36.8, 36.7, 32.8, 25.9 (3C), 18.2, 17.1, -5.5 (2C); MS (CI), $m / z$ (relative intensity) 484 ([M+H $\left.\left.{ }^{+}\right], 85 \%\right)$; Anal. Calcd for $\mathrm{C}_{28} \mathrm{H}_{42} \mathrm{~N}_{2} \mathrm{O}_{3} \mathrm{Si}$ : C, 69.67; $\mathrm{H}$, 8.77; N, 5.80. Found: C, 69.59; H, 8.75; N, 5.75.

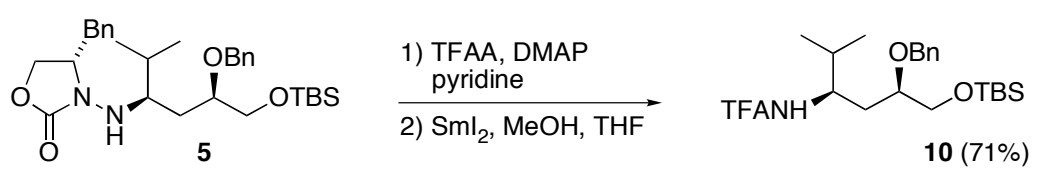

$(\boldsymbol{R}, \boldsymbol{R})$-Trifluoroacetamide 10 (General Procedure C). Trifluoroacetic anhydride (TFAA, $2.58 \mathrm{~mL}$ ) was added to a solution of the hydrazine $5(136 \mathrm{mg}, 0.26 \mathrm{mmol})$ and DMAP (4 mg, $0.03 \mathrm{mmol}) \mathrm{in} \mathrm{NEt}_{3}$ $(360 \mu \mathrm{L})$ at $0{ }^{\circ} \mathrm{C}$. After two hours, the reaction mixture was allowed to warm to ambient temperature and stirred overnight. After cooling to $0{ }^{\circ} \mathrm{C}$, sat. $\mathrm{NaHCO}_{3}$ was added dropwise under vigorous stirring and the resulting mixture was partitioned between EtOAc and brine, then the organic phase was dried with $\mathrm{MgSO}_{4}$. Concentration and flash chromatography (Hex $\rightarrow 3 / 1$ Hex:EtOAc) afforded the trifluoroacetohydrazide as a pale yellow gum (115 mg, $71 \%$ yield); a mixture of rotamers exhibiting complicated NMR spectra of limited utility; $[\alpha]_{\mathrm{D}}^{20}+43.6\left(c 1.65, \mathrm{CHCl}_{3}\right)$; IR (film) 2971, 2925, 2883, 1786, 1716, 1496, 1473, 1456, 1399, 1350, 1250, 1215, 1160, 1113, 835, 772, 736, $699 \mathrm{~cm}^{-1}$; Anal. Calcd for $\mathrm{C}_{32} \mathrm{H}_{45} \mathrm{~F}_{3} \mathrm{~N}_{2} \mathrm{O}_{5} \mathrm{Si}$ : C, 61.71; H, 7.28; N, 4.50. Found: C, 62.10; H, 7.42; N, 4.39.

To a solution of the trifluoroacetohydrazide obtained as described above (115 $\mathrm{mg}, 0.18 \mathrm{mmol})$ in THF/MeOH (1:1 v/v, $0.68 \mathrm{~mL}$ ) was added dropwise a freshly prepared solution of $\mathrm{SmI}_{2}$ in $\mathrm{THF}^{5}$ (ca. 0.3 M) until the blue color remained. After quenching the reaction mixture by opening to air, filtration through silica gel $\left(\mathrm{Et}_{2} \mathrm{O}\right.$ eluent), concentration, and flash chromatography afforded the protected trifluoroacetamide $\mathbf{1 0}$ as a pale yellow oil $(82 \mathrm{mg}, 100 \%$ yield $) ;[\alpha]_{\mathrm{D}}^{20}+9.6\left(c 3.50, \mathrm{CHCl}_{3}\right)$; IR (film) 3314, 2958, 2929, 2858, 1704, 1550, 1472, 1390, 1361, 1253, 1205, 1180, 1161, 1115, 1093, 837, 777, 724, $697 \mathrm{~cm}^{-1} ;{ }^{1} \mathrm{H}$ NMR $\left(500 \mathrm{MHz}, \mathrm{CDCl}_{3}\right) \delta 7.38-7.31(\mathrm{~m}, 5 \mathrm{H}), 6.92(\mathrm{br} \mathrm{d}, J=8.5 \mathrm{~Hz}, 1 \mathrm{H}), 4.74(\mathrm{~d}, J=$ $11.0 \mathrm{~Hz}, 1 \mathrm{H}), 4.50(\mathrm{~d}, J=11.0 \mathrm{~Hz}, 1 \mathrm{H}), 3.95(\mathrm{qd}, J=7.8,2.5 \mathrm{~Hz}, 1 \mathrm{H}), 3.76(\mathrm{dd}, J=10.2,4.8 \mathrm{~Hz}, 1 \mathrm{H})$, $3.63(\mathrm{~m}, 1 \mathrm{H}), 3.56(\mathrm{dd}, J=10.2,5.8 \mathrm{~Hz}, 1 \mathrm{H}), 1.83(\mathrm{ddd}, J=14.9,8.1,2.7 \mathrm{~Hz}, 1 \mathrm{H}), 1.75(\mathrm{~m}$, apparent octet, $J=6.9 \mathrm{~Hz}, 1 \mathrm{H}), 1.70(\mathrm{ddd}, J=14.8,10.0,2.7 \mathrm{~Hz}, 1 \mathrm{H}), 0.93(\mathrm{~d}, J=6.8 \mathrm{~Hz}, 3 \mathrm{H}), 0.91(\mathrm{~s}, 9 \mathrm{H}), 0.89$ $(\mathrm{d}, J=6.7 \mathrm{~Hz}, 3 \mathrm{H}), 0.08(\mathrm{~s}, 6 \mathrm{H}) ;{ }^{13} \mathrm{C} \mathrm{NMR}\left(125 \mathrm{MHz}, \mathrm{CDCl}_{3}\right) \delta 156.7(\mathrm{~J}=36 \mathrm{~Hz}), 137.9,128.5(2 \mathrm{C})$, $128.2(2 \mathrm{C}), 127.9,116.0(\mathrm{~J}=275 \mathrm{~Hz}), 77.2,72.8,64.9,53.1,33.2,31.3,25.8(3 \mathrm{C}), 19.0,18.6,18.1,-5.5$ (2C); Anal. Calcd for $\mathrm{C}_{22} \mathrm{H}_{36} \mathrm{~F}_{3} \mathrm{NO}_{3} \mathrm{Si}$ : C, 59.03; H, 8.11; N, 3.13. Found: C, 59.32; H, 8.21; N, 3.14.
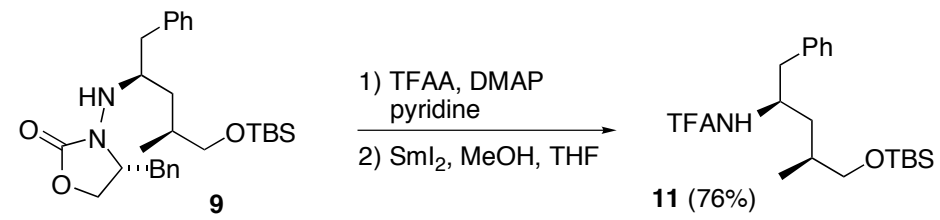

Trifluoroacetamide 11. From the hydrazine $9(94 \mathrm{mg}, 0.19 \mathrm{mmol})$, following the General Procedure (C), the intermediate trifluoroacetohydrazide was obtained as a pale yellow oil (89 $\mathrm{mg}$, 79\% yield); a mixture of rotamers exhibiting complicated NMR spectra of limited utility; $[\alpha]_{\mathrm{D}}^{20}-10.4\left(c 0.25, \mathrm{CHCl}_{3}\right)$; IR (film) 2956, 2930, 2856, 1791, 1717, 1653, 1559, 1456, 1398, 1359, 1255, 1209, 1193, 1174, 1159, $1107,1032,839,775,735,701 \mathrm{~cm}^{-1}$; MS (CI), $\mathrm{m} / \mathrm{z}$ (relative intensity) $580\left(\left[\mathrm{M}+\mathrm{H}^{+}\right], 5 \%\right)$.

Treatment of the trifluoroacetohydrazide $\left(77 \mathrm{mg}, 0.13 \mathrm{mmol}\right.$ ) with $\mathrm{SmI}_{2}$ as described in General Procedure (C) afforded trifluoroacetamide 11 as a colorless solid (52 mg, 97\% yield); mp 87-89 ${ }^{\circ} \mathrm{C}$ (from 99/1 Hex:Et ${ }_{2} \mathrm{O}$ ); $[\alpha]_{\mathrm{D}}^{20}+7.5\left(c 5.40, \mathrm{CHCl}_{3}\right.$ ); IR (film) 3341, 2955, 2930, 2857, 1700, 1551, 1496, 1472 , 1461, 1389, 1362, 1251, 1230, 1201, 1182, 1160, 1111, 1074, 1045, 993, 865, 839, 774, 756, 705, 
$667 \mathrm{~cm}^{-1} ;{ }^{1} \mathrm{H}$ NMR $\left(500 \mathrm{MHz}, \mathrm{CDCl}_{3}\right) \delta$ 7.32-7.16 (m, 5H), 6.66 (br d, J= 7.3 Hz, 1H), 4.34-4.27 (m, $1 \mathrm{H}), 3.56(\mathrm{dd}, J=10.2,4.8 \mathrm{~Hz}, 1 \mathrm{H}), 3.38(\mathrm{dd}, J=10.2,6.8 \mathrm{~Hz}, 1 \mathrm{H}), 2.91-2.83(\mathrm{~m}, 2 \mathrm{H}), 1.83-1.74(\mathrm{~m}$, $1 \mathrm{H}), 1.69$ (ddd, $J=14.1,7.4,5.0 \mathrm{~Hz}, 1 \mathrm{H}), 1.42(\mathrm{ddd}, J=14.1,8.4,5.7 \mathrm{~Hz}, 1 \mathrm{H}), 0.91-0.88(\mathrm{~m}, 12 \mathrm{H})$, $0.06(\mathrm{~s}, 6 \mathrm{H}) ;{ }^{13} \mathrm{C}$ NMR $\left(125 \mathrm{MHz}, \mathrm{CDCl}_{3}\right) \delta 156.5(\mathrm{~J}=36 \mathrm{~Hz}), 136.8,129.3(2 \mathrm{C}), 128.5(2 \mathrm{C}), 126.7$, $115.8(J=287 \mathrm{~Hz}), 70.3,67.3,49.3,40.0,37.0,32.4,25.8(3 \mathrm{C}), 18.3,17.2,-5.5$, -5.6. Anal. Calcd for $\mathrm{C}_{20} \mathrm{H}_{32} \mathrm{~F}_{3} \mathrm{NO}_{2} \mathrm{Si}: \mathrm{C}, 59.52 ; \mathrm{H}, 7.99 ; \mathrm{N}, 3.47$. Found: C, 59.58; H, 8.07; N, 3.39.

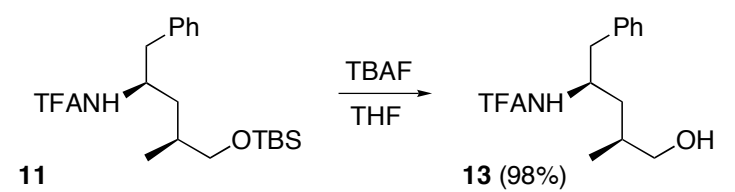

Alcohol 13 (General Procedure D). To a solution of silyl ether 11 (108 mg, $0.27 \mathrm{mmol}$ ) in dry THF $(5.35 \mathrm{~mL})$ was added dropwise a solution of TBAF $(1.0 \mathrm{M}$ in THF, $0.40 \mathrm{~mL}, 0.40 \mathrm{mmol})$ at ambient temperature. After 30 minutes the reaction mixture was poured into sat. $\mathrm{NH}_{4} \mathrm{Cl}$. The organic phase was washed with brine and dried over $\mathrm{MgSO}_{4}$. Concentration and flash chromatography (Hex $\rightarrow 1 / 2$ Hex:EtOAc) afforded the alcohol 13 as a colorless solid $(76 \mathrm{mg}, 98 \%$ yield $) ; \mathrm{mp} 79-80^{\circ} \mathrm{C} ;[\alpha]_{\mathrm{D}}^{20}+11.8$ (c 3.90, $\mathrm{CHCl}_{3}$ ); IR (film) 3405, 3292, 3089, 3031, 2961, 2927, 2877, 1700, 1653, 1575, 1568, 1559, 1540, 1497, 1456, 1374, 1206, 1187, 1162, 1032, 871, 747, 725, $700 \mathrm{~cm}^{-1} ;{ }^{1} \mathrm{H}$ NMR (500 MHz, $\left.\mathrm{CDCl}_{3}\right)$ d 7.33-7.17 (m, 5H), $6.94(\mathrm{br} \mathrm{d}, J=5.1 \mathrm{~Hz}, 1 \mathrm{H}), 4.39-4.32(\mathrm{~m}, 1 \mathrm{H}), 3.62(\mathrm{dd}, J=10.4,4.9 \mathrm{~Hz}, 1 \mathrm{H}), 3.43$ $(\mathrm{dd}, J=10.4,7.3 \mathrm{~Hz}, 1 \mathrm{H}), 2.92-2.84(\mathrm{~m}, 2 \mathrm{H}), 1.87-1.80(\mathrm{~m}, 1 \mathrm{H}), 1.67(\mathrm{ddd}, J=14.4,7.9,4.7 \mathrm{~Hz}, 1 \mathrm{H})$, $1.48(\mathrm{ddd}, J=14.4,8.0,4.9 \mathrm{~Hz}, 1 \mathrm{H}), 0.92(\mathrm{~d}, J=6.9 \mathrm{~Hz}, 3 \mathrm{H}) ;{ }^{13} \mathrm{C} \mathrm{NMR}\left(125 \mathrm{MHz}, \mathrm{CDCl}_{3}\right) \delta 156.8(J=$ $36 \mathrm{~Hz}), 136.9,129.2(2 \mathrm{C}), 128.6(2 \mathrm{C}), 126.8,115.9(\mathrm{~J}=286 \mathrm{~Hz}), 67.7,49.6,39.9,37.8,31.8$, 17.5. Anal. Calcd for $\mathrm{C}_{14} \mathrm{H}_{18} \mathrm{~F}_{3} \mathrm{NO}_{2}$ : C, 58.12; H, 6.27; N, 4.84. Found: C, 58.36; H, 6.30; N, 4.76.

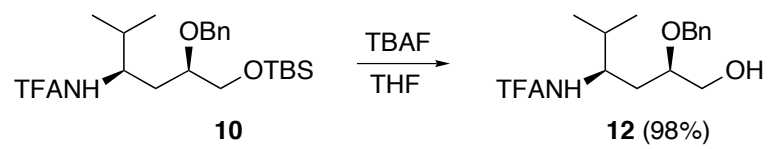

Alcohol 12. From the silyl ether $10(82 \mathrm{mg}, 0.18 \mathrm{mmol})$ following the General Procedure (D), the alcohol 12 was obtained as a colorless oil $(60 \mathrm{mg}, 98 \%$ yield $) ;[\alpha]_{\mathrm{D}}^{20}+15.1\left(c 4.4, \mathrm{CHCl}_{3}\right)$; IR (film) 3431, 3306, 3091, 3033, 2965, 2931, 2877, 1700, 1558, 1497, 1466, 1455, 1392, 1372, 1347, 1207, 1185, 1157, 1088, 1047, 1028, 922, 880, 726, $698 \mathrm{~cm}^{-1} ;{ }^{1} \mathrm{H}$ NMR $\left(500 \mathrm{MHz}, \mathrm{CDCl}_{3}\right) \delta 7.37-7.31(\mathrm{~m}$, $5 \mathrm{H}), 6.77(\mathrm{br} \mathrm{d}, J=8.4 \mathrm{~Hz}, 1 \mathrm{H}), 4.58(\mathrm{ABq}, J=11.1 \mathrm{~Hz}, \Delta v=51.0 \mathrm{~Hz}, 2 \mathrm{H}), 4.03-3.97(\mathrm{~m}, 1 \mathrm{H}), 3.77$ (dd, $J=11.3,4.2 \mathrm{~Hz}, 1 \mathrm{H}), 3.63-3.59(\mathrm{~m}, 1 \mathrm{H}), 3.56$ (dd, $J=11.3,3.8 \mathrm{~Hz}, 1 \mathrm{H}), 2.00$ (br s, $1 \mathrm{H}), 1.89$ (ddd, $J=15.0,9.8,2.8 \mathrm{~Hz}, 1 \mathrm{H}), 1.78(\mathrm{~m}$, apparent octet, $J=6.9 \mathrm{~Hz}, 1 \mathrm{H}), 1.69(\mathrm{ddd}, J=15.0,8.9,3.1 \mathrm{~Hz}$, $1 \mathrm{H}), 0.93(\mathrm{~d}, J=6.8 \mathrm{~Hz}, 3 \mathrm{H}), 0.90(\mathrm{~d}, J=6.7 \mathrm{~Hz}, 3 \mathrm{H}) ;{ }^{13} \mathrm{C} \mathrm{NMR}\left(125 \mathrm{MHz}, \mathrm{CDCl}_{3}\right) \delta 156.9(J=36$ $\mathrm{Hz}), 134.6,128.6(2 \mathrm{C}), 128.1(3 \mathrm{C}), 116.0(\mathrm{~J}=286 \mathrm{~Hz}), 76.7,72.4,63.6,52.8,33.3,31.7,18.9$, 18.3; Anal. Calcd for $\mathrm{C}_{16} \mathrm{H}_{22} \mathrm{~F}_{3} \mathrm{NO}_{3}$ : C, 57.65; H, 6.65; N, 4.20. Found: C, 57.66; H, 6.75; N, 4.18.

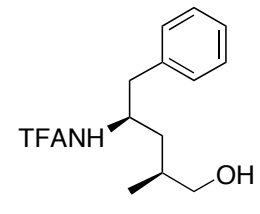

13

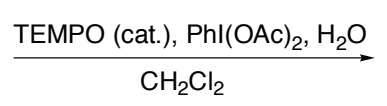

$\boldsymbol{\gamma}$-Amino acid B. The alcohol $13(22 \mathrm{mg}, 0.076 \mathrm{mmol})$ was dissolved in $\mathrm{CH}_{2} \mathrm{Cl}_{2}(1 \mathrm{~mL})$. Water (ca. $0.2 \mathrm{~mL})$ was added, followed by $\mathrm{PhI}(\mathrm{OAc})_{2}(54 \mathrm{mg}, 0.17 \mathrm{mmol})$ and TEMPO $(1.2 \mathrm{mg}, 0.0076 \mathrm{mmol})$ were added in this order at $0{ }^{\circ} \mathrm{C}$ and the mixture was warmed to ambient temperature over $30 \mathrm{~min}$. The 
aldehyde intermediate was present. ${ }^{6}$ Additional TEMPO $(0.6 \mathrm{mg})$ was added. After $12 \mathrm{~h}$, the mixture was diluted with $\mathrm{Et}_{2} \mathrm{O}(25 \mathrm{~mL})$ and extracted with sat. $\mathrm{NaHCO}_{3}(2 \times 25 \mathrm{~mL})$. The aqueous phase was acidified with $2 \mathrm{M} \mathrm{HCl}$ and extracted with $\mathrm{CH}_{2} \mathrm{Cl}_{2}(3 \times 100 \mathrm{~mL})$. This organic phase was washed with brine and dried over $\mathrm{MgSO}_{4}$. Concentration afforded carboxylic acid $\mathbf{B}$ as a colorless solid (19 mg, 83\% yield); mp 119-120 ${ }^{\circ} \mathrm{C}$ (from 9:1 Hex/Et $2 \mathrm{O}$ ); $[\alpha]_{\mathrm{D}}^{20}+10.3$ (c 0.75, $\mathrm{CHCl}_{3}$ ); IR (film) 3302, 3091, 3067, 3031, 2978, 2926, 2661, 1712, 1701, 1558, 1496, 1456, 1373, 1286, 1248, 1205, 1188, 1162, 1085, 939, 869, 754, 724, $702 \mathrm{~cm}^{-1} ;{ }^{1} \mathrm{H}$ NMR $\left(500 \mathrm{MHz}, \mathrm{CDCl}_{3}\right) \delta 10.6$ (br s, 1H), 7.33-7.24 (m, 4H), 7.16 (m, $\left.1 \mathrm{H}\right)$, 6.35 (br d, $J=8.1 \mathrm{~Hz}, 1 \mathrm{H}), 4.32-4.25(\mathrm{~m}, 1 \mathrm{H}), 2.94-2.88(\mathrm{~m}, 2 \mathrm{H}), 2.66-2.58(\mathrm{~m}, 1 \mathrm{H}), 1.99(\mathrm{ddd}, J=$ $14.5,9.3,4.0 \mathrm{~Hz}, 1 \mathrm{H}), 1.72(\mathrm{ddd}, J=14.5,9.9,4.1 \mathrm{~Hz}, 1 \mathrm{H}), 1.21(\mathrm{~d}, J=7.2 \mathrm{~Hz}, 3 \mathrm{H}) ;{ }^{13} \mathrm{C}$ NMR $(125$ $\left.\mathrm{MHz}, \mathrm{CDCl}_{3}\right) \delta 181.1,156.8(\mathrm{~J}=37 \mathrm{~Hz}), 136.2,129.2(2 \mathrm{C}), 128.7(2 \mathrm{C}), 127.0,115.7(J=287 \mathrm{~Hz})$, 49.5, 40.2, 36.4, 35.9, 17.0. Anal. Calcd for $\mathrm{C}_{14} \mathrm{H}_{16} \mathrm{~F}_{3} \mathrm{NO}_{3}$ : C, 55.44; H, 5.32; N, 4.62. Found: C, 55.31; $\mathrm{H}, 5.37 ; \mathrm{N}, 4.56$.

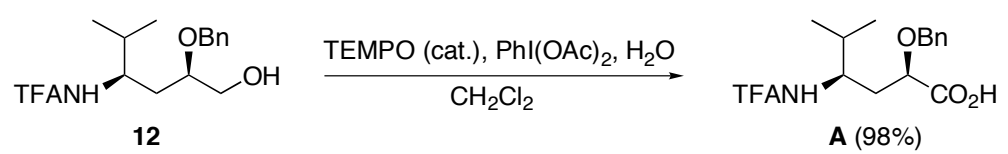

$\boldsymbol{\gamma}$-Amino acid A. From the alcohol $12(40 \mathrm{mg}, 0.12 \mathrm{mmol})$, the procedure for preparation of $\mathbf{B}$ was employed, using one drop of water and $5 \mathrm{~mol} \%$ TEMPO. The carboxylic acid $\mathbf{A}$ was obtained as a white solid (40 mg, 98\% yield); mp $113-115^{\circ} \mathrm{C}$ (from 90:10 $\mathrm{Hex}_{2} \mathrm{Et}_{2} \mathrm{O}$ ); $[\alpha]_{\mathrm{D}}^{20}+34.5\left(c 0.32, \mathrm{CHCl}_{3}\right.$ ); IR (film) 3304, 3094, 3034, 2965, 2926, 2876, 2855, 2666, 1716, 1700, 1558, 1540, 1472, 1436, 1394, 1373, 1333, 1208, 1184, 1164, 1117, 1028, 914, 882, 805, 736, 720, $698 \mathrm{~cm}^{-1}$; ${ }^{1} \mathrm{H}$ NMR $(500 \mathrm{MHz}$, $\left.\mathrm{CDCl}_{3}\right) \delta 7.38-7.34(\mathrm{~m}, 5 \mathrm{H}), 6.63(\mathrm{br} \mathrm{d}, J=9.2 \mathrm{~Hz}, 1 \mathrm{H}), 4.72(\mathrm{~d}, J=10.7 \mathrm{~Hz}, 1 \mathrm{H}), 4.43(\mathrm{~d}, J=10.7 \mathrm{~Hz}$ $1 \mathrm{H}), 4.10-4.01(\mathrm{~m}, 2 \mathrm{H}), 2.09-2.00(\mathrm{~m}, 2 \mathrm{H}), 1.75(\mathrm{~m}$, apparent octet, $J=6.8 \mathrm{~Hz}, 1 \mathrm{H}), 0.94(\mathrm{~d}, J=6.7 \mathrm{~Hz}$, $3 \mathrm{H}), 0.91(\mathrm{~d}, J=6.8 \mathrm{~Hz}, 3 \mathrm{H})$ (carboxylic acid $\mathrm{H}$ was not visible) ${ }^{13} \mathrm{C} \mathrm{NMR}\left(125 \mathrm{MHz}, \mathrm{CDCl}_{3}\right) \delta 176.2$, $157.3(\mathrm{~J}=36 \mathrm{~Hz}), 136.2,128.6(4 \mathrm{C}), 128.5,115.9(\mathrm{~J}=286 \mathrm{~Hz}), 75.0,73.6,52.5,34.4,31.6,18.8$, 18.2. Anal. Calcd for $\mathrm{C}_{16} \mathrm{H}_{20} \mathrm{~F}_{3} \mathrm{NO}_{4}: \mathrm{C}, 55.33 ; \mathrm{H}, 5.80 ; \mathrm{N}, 4.03$. Found: C, 55.45; H, 5.84; N, 3.97.

(1) The compound $(S)-\mathbf{3}$ has been previously reported. For characterizations, see Friestad, G. K.; Qin, J. J. Am. Chem. Soc. 2000, 122, 8329-8330.

(2) The preparation, starting from $(R)$-4-phenylmethyl-2-oxazolidinone, and all spectral characteristics of $(R)-\mathbf{3}$ are the same as previously described (see reference 1 ) except for the optical rotation: $(R)-3:[\alpha]_{\mathrm{D}}^{20}-72.5\left(c 3.48, \mathrm{CHCl}_{3}\right)$

(3) All the characterizations of the iodide 7 are consistent with Nakamura, Y.; Mori, K. Eur. J. Org. Chem. 2000, 2745-2753.

(4) The photolysis reaction was performed in a Schlenk tube (Pyrex) equipped with a cold finger. The $\mathrm{CH}_{2} \mathrm{Cl}_{2}$ was degassed vigorously $30 \mathrm{~min}$ with dry oxygen-free nitrogen prior to use. The hydrazone was dried three times using benzene azeotrope distillation and put under vacuum for 1 $\mathrm{h}$. The indium chloride (Aldrich) was dried at least $2 \mathrm{~h}$ under high vacuum at room temperature.

(5) For the preparation of the $\mathrm{SmI}_{2}$ solution in THF, see Ding, H.; Friestad, G. K. Org. Lett. 2004, 6, 637-640.

(6) During preparation of $\mathbf{B}$, the aldehyde intermediate was isolated and identified by reduction to alcohol $13\left(\mathrm{NaBH}_{4}\right)$. The analogous aldehyde was not observed during preparation of $\mathbf{A}$. 
Supporting Information - Friestad et al.

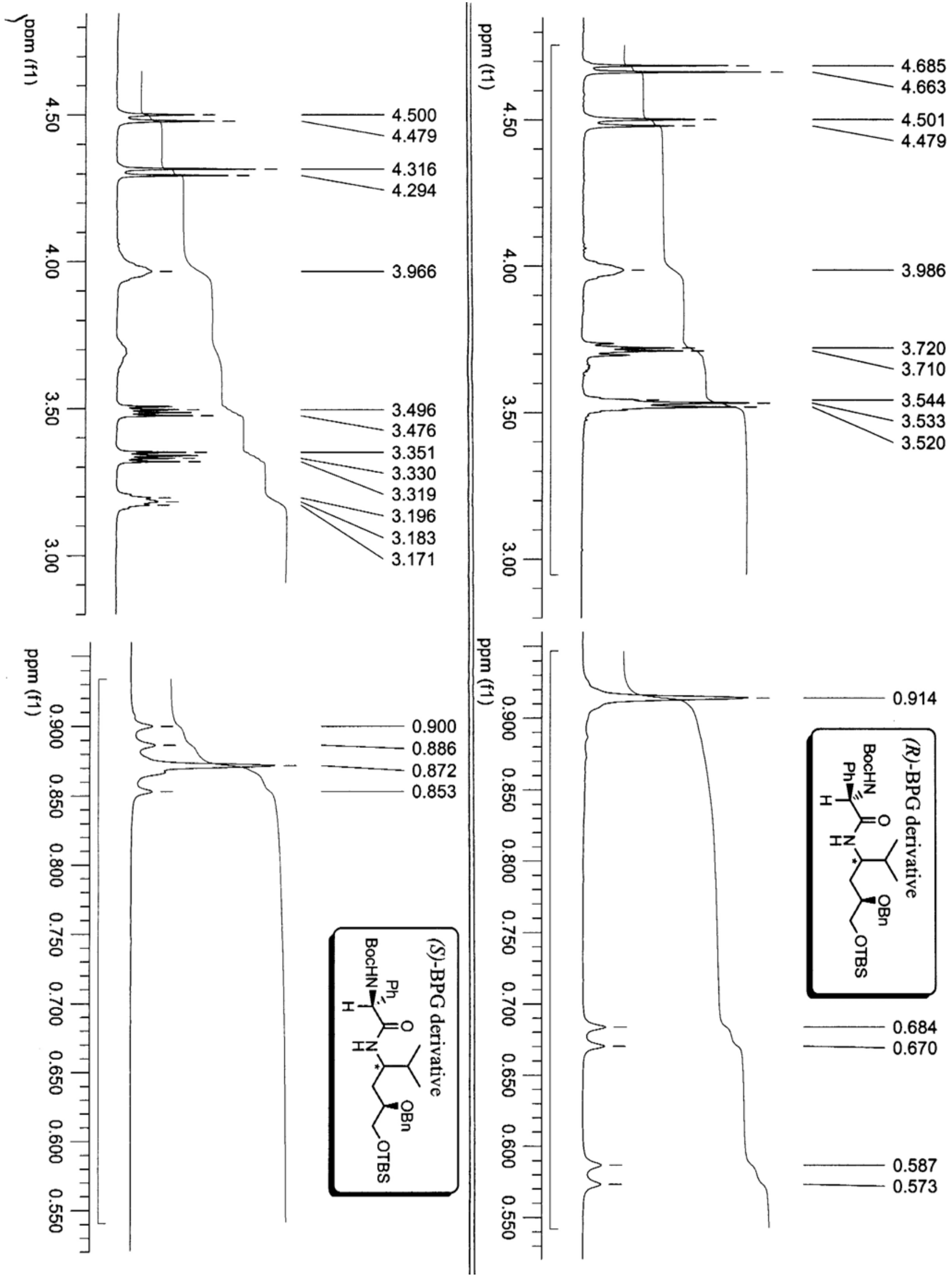

